\title{
INFINITE PRODUCTS OVER VISIBLE LATTICE POINTS
}

GEOFFREY B. CAMPBELL

Mathematics Research Section

Institute of Advanced Studies

School of Mathematical Sciences

The Australian National University

GPO Box 4, Canberra, Australia, 2601

(Received August 10, 1992 and in revised form September 2, 1993)

\begin{abstract}
About fifty new multivariate combinatorial identities are given, connected with partition theory, prime products, and Dirichlet series. Connections to Lattice Sums in Chemistry and Physics are alluded to also.
\end{abstract}

KEY WORDS AND PHRASES: Combinatorial Identities, Lattice Points in Specified Regions, Riemann's Zeta Function, Dirichlet Series and other series expansions.

1991 AMS SUBJECT CLASSIFICATION CODES: 05A19, 11P21, 11M06, 30B50.

\section{INTRODUCTION}

The concept of visible-from-the-origin lattice points in the $X-Y$ plane was described in Apostol [2], where their density in the space was calculated. Visible from the origin lattice points have the property that their coordinates are relatively prime. Recently, a host of new identities were found, based on summation or products taken on these points (see Campbell [4], [5], [6]). We present here some of the more notable results from these three papers, then extend them to higher dimensions giving general theorems accordingly.

Firstly, we have the three "companion identities," so called because of their interdependence, and their relation to the visible from origin lattice points in the first quadrant. We use the operators defined by

$$
{ }_{k} \sum_{j}=\sum_{k=2}^{\infty} \sum_{j \in \phi_{k}}, \quad \Pi_{k}=\prod_{k=2}^{\infty} \prod_{j \in \phi_{k}}
$$

where $\phi_{k}$ is the set of positive integers less than and relatively prime to $k$.

The three "companions" given in [4], [5], and [6] are

$$
\begin{gathered}
(1-y)_{k} \Pi_{j}\left(1-x^{j} y^{k}\right)^{1 / k}=\left(\frac{1-y}{1-x y}\right)^{1 / 1-x)}, \quad(x \neq 1) \\
(1-x y)_{k} \Pi_{j}\left(1-x^{k} y^{\prime}\right)^{1 / j}=(1-x y)^{1 / 1-x)}, \\
(1-y)(1-x y)_{k} \Pi_{j}\left(1-x^{J} y^{k}\right)^{1 / k}\left(1-x^{k} y^{J}\right)^{1 / j}=(1-y)^{1 / 1-x)},
\end{gathered}
$$

where convergence occurs respectively for $|x y|$ and $|y|<1,|x|$ and $|x y|<1,|x|$ and $|y|<1$. It is worth noting that the left side of (1.3) can be rewritten

$$
(1-y) \prod_{\substack{(a, b)=1 \\ a, b \in z^{+}}}\left(1-x^{a} y^{b}\right)^{1 / b},
$$

where $(a, b)$ denotes the greatest common divisor of $a$ and $b$. A generalization of this gcd idea will be applied later in this paper. 
It is clear that the identities (1.1), (1.2) and (1.3) belong naturally together when we see that any one of them implies the other two. The reader can easily verify by iterative multiplication on (1.1) that $x y, x^{2} y, \ldots$ substituted for $y$ leads to (1.3). (1.3) then divided on both sides by (1.1) gives next (1.2). If $y / x$ is substituted for $y$ in (1.2) we obtain (1.3).

Later we shall prove generalizations of (1.1) to (1.3) both in two dimensions and in higher dimensions. The "companion identity" idea is not restricted to just the above formulae. In [6], for example, the new products taken over prime numbers $p$ were given as follows

$$
\begin{gathered}
\zeta(n)_{k} \Pi_{j} \zeta(j m+k n)^{1 / k}=\prod_{p}\left(\frac{1-p^{-(m+n)}}{1-p^{-n}}\right)^{1 / 1-p-m)}, \\
\zeta(m+n)_{k} \Pi_{j} \zeta(k m+j n)^{1 / j}=\prod_{p}\left(1-p^{-(m+n)}\right)^{-1 / 1-p-m)}, \\
\zeta(n) \zeta(m+n)_{k} \Pi_{j} \zeta(j m+k n)^{1 / k} \zeta(k m+j n)^{1 / j}=\prod_{p}\left(1-p^{-n}\right)^{-1 / 1-p-m)},
\end{gathered}
$$

valid with $p^{m} \neq 1$ for respectively $\operatorname{Re} m+n$ and $\operatorname{Re} n>1, \operatorname{Re} m$ and $\operatorname{Re} m+n>1, \operatorname{Re} m$ and $\operatorname{Re} n>1$. These identities were derived from (1.1) to (1.3) by substitution of $x=p^{-m}, y=p^{-n}$, and forming the product over primes $p$ on each side. (1.4) to (1.6) led to results concerning the multiplicative function

$$
\omega(m, k)=\prod_{i=1}^{s}\left(\begin{array}{c}
1 /\left(1-p_{i}^{-m}\right) \\
a_{i}
\end{array}\right) \text { with } k=\prod_{i=1}^{s} p_{i}^{a_{i}}
$$

As with the products (1.1) to (1.3), we shall give generalized versions of (1.4) to (1.6), or at least methods for doing so.

The underlying theorems for the products (1.1) to (1.3) were

THEOREM 1.1. If $\left(a_{k}\right)$ and $\left(b_{k}\right)$ are sequences of functions chosen so the following series converge, then

$$
\sum_{k=1}^{\infty} a_{k} \frac{1-\exp \left(b_{k} q\right)}{1-\exp \left(b_{k} q / k\right)}=\sum_{k=1}^{\infty} S_{k}
$$

where $S_{k}$ is defined by

$$
S_{k}= \begin{cases}\sum_{j=1}^{\infty} a_{j}, & k=1, \\ \sum_{j=1}^{\infty} a_{j k} \sum_{h \in \phi_{k}} \exp \left(b_{j k} h q / k\right), & k>1,\end{cases}
$$

and $\phi_{k}$ is the set of positive integers less than and relatively prime to $k$.

For a proof of this see Campbell [4]. (1.1) derives from the case of the theorem where $a_{k}=y^{k} k^{-1}$, $b_{k}=k \log x q^{-1}$. Secondly, a finite version of Theorem 1.1 was given in Campbell [5].

THEOREM 1.2. If $\left(a_{k}\right)$ and $\left(b_{k}\right)$ are arbitrary sequences chosen so that, together with choice of $x$, the following functions are all defined then

$$
\sum_{k=1}^{n} a_{k} \frac{1-\exp \left(b_{k} x\right)}{1-\exp \left(b_{k} x / k\right)}=\left\{\sum_{k=1}^{n} a_{j}\right\}+\sum_{m=2}^{n} \sum_{k=1}^{[n / m]} \sum_{j \in \phi_{m}} a_{m k} \exp \left(b_{m k} j x / m\right)
$$

where $[\boldsymbol{n}]$ denotes the greatest integer in $\boldsymbol{n}$.

Each theorem above is equivalent, and each theorem has advantages over the other. Theorem 1.1 is easier to grasp and apply in a range of situations, and Theorem 1.2 is better for examining convergence 
questions associated with the substituted functions as $n$ is permitted to increase. Either theorem could be used (although Theorem 1.2 was applied in Campbell [5]) to obtain new identities for Jacobi theta functions. The diversity of, say (1.10), is that it yields also proofs of classical results such as

$$
\sigma_{-s}(n) \zeta(s+1)^{-1}=\sum_{k=1} c_{k}(n) k^{-s-1}, \quad \operatorname{Re} s>1
$$

due to Ramanujan, where $\sigma_{s}(n)$ is the sum of $\operatorname{sth}$ powers of the divisors of $n$, and $c_{k}(n)$ is the multiplicative function

$$
c_{k}(n)-\sum_{, \phi_{k}} \cos \left(\frac{2 \pi n j}{k}\right) .
$$

Also, in [5], further results were obtained, such as

$$
\prod_{k=1}^{\infty}\left(1-y^{k}\right)^{-c_{k}(n) k k}=\exp \left\{\sum_{r} y^{r}\right\}, \quad|y|<1,
$$

and, the rather unusual formula for $|q|<1$,

$$
\begin{aligned}
& \frac{\theta_{1}(\alpha+\beta) \sin (\alpha-\beta)}{\theta_{1}(\alpha-\beta) \sin (\alpha+\beta)} \prod_{k=1}^{\infty}\left(\frac{\theta_{1}\left((\alpha+\beta) k, q^{2 k}\right) \sin (\alpha-\beta)}{\theta_{1}\left((\alpha-\beta) k, q^{2 k}\right) \sin (\alpha+\beta)}\right) c_{k}(n) / k \\
& =\exp \left\{4 \sum_{k=1} \frac{q^{2 k}}{1-q^{2 k}} \sin 2 k \alpha \sin 2 k \beta\right\}
\end{aligned}
$$

in which

$$
\theta_{1}(z, q)=\theta_{1}(z)=2 q^{\frac{1}{4}} \sum_{k=0}^{\infty}(-1)^{k} q^{k(k+1)} \sin (2 k+1) z .
$$

Evidently there is scope for much research on these types of indentities, and whilst Theorems 1.1 and 1.2 may have easily stated generalizations in higher dimensions, or even "companions" in the sense of (1.1), (1.2), and (1.3), in this paper we shall specialize although still in higher dimensions, and obtain numerous new and simple identities.

\section{AN INFINITE PRODUCT THEOREM IN $N$-SPACE}

In this section we give a general theorem on multivariate infinite products and a method for obtaining an infinite variety of similar theorems. The drawback from this is that most of the other obtainable theorems will require extra effort to work out. The ideas regarding companion identities as stated in $§ 1$ may help us to simply determine whole classes of interdependent formulae in higher dimensional Euclidean space. We use the notation $\left(a_{1}, a_{2}, \ldots, a_{n}\right)$ to mean "the greatest common divisor of all of $a_{1}, a_{2}, \ldots, a_{n}$ together." It will be important to distinguish between this and the ordered $n$-tuple given by the vector notation $\left\langle a_{1}, a_{2}, \ldots, a_{n}\right\rangle$. In either case we will be concerned with lattice points in the relevant space, hence any vector or gcd will be over integer coordinates. The infinite product (1.3) is a particular case of the

THEOREM 2.1. If $i=1,2, \ldots, n$, then for each $\left|x_{\imath}\right|<1$, and $b_{i} \in \mathbf{C}$,

$$
\begin{aligned}
& \prod_{\substack{\left(a_{1}, a_{2}, \ldots, a_{n}\right)-1 \\
a_{1} \in z^{+}}}\left(1-x_{1}^{a_{1}} x_{2}^{a_{2}} \ldots x_{n}^{a_{n}}\right)^{\frac{a_{1} a_{1} a_{2}^{b_{2}} \cdot a_{n}^{b_{n}}}{n_{n}}} \\
& \quad=\exp \left\{\prod_{i=1}^{n}\left(\sum_{,=1}^{\infty} \frac{x_{i}^{\prime}}{j^{b_{1}}}\right)\right\}, \text { provided } \sum_{i=1}^{n} b_{i}=1 .
\end{aligned}
$$

Before proving this we state a definition and prove a lemma. These will be re-used in later work. 
DEFINITION 2.2. Any Euclidean vector $\left\langle a_{1}, a_{2}, \ldots, a_{n}\right\rangle$ for which $\left(a_{1}, a_{2}, \ldots, a_{n}\right)=1$ we call a visible point vector, abbreviated vpv.

LEMMA 2.3. Consider an infinite region raying out of the origin in any Euclidean vector space. The set of all lattice point vectors apart from the origin in that region is precisely the set of positive integer multiples of the visible point vectors (vpv's) in that region.

PROOF. Each vpv will have integer coordinates whose greatest common divisor is unity. Viewed from the origin, all other lattice points are obscured behind the vpv end points. If $x$ is a vpv in the region then all vectors in that region from the origin with direction of $x$ preserved are enumerated by a sequence $1 x, 2 x, 3 x, \ldots$, and the greatest common divisor of the components of $n x$ is clearly $n$. This is because if the scalar $n$ is non-integer at least one of the coordinates of $n x$ would be non-integer. Therefore, if the vpv's in the region are countably given by $x_{1}, x_{2}, x_{3}, \ldots$, then all lattice point vectors from the origin in the region are

$$
1 x_{1}, 2 x_{1}, 3 x_{1}, \ldots, 1 x_{2}, 2 x_{2}, 3 x_{2}, \ldots, 1 x_{3}, 2 x_{3}, 3 x_{3}, \ldots \text {, etc. . }
$$

Completion of the proof comes with recognition that the set of all vpv's in any "rayed from the origin" region in any Euclidean vector space is a countable set. Proof of this last assertion is by induction on the dimension, knowing that the lattice points are countable in any two dimensional region. As we count each lattice point vector in the desired region we decide whether it is a vpv simply by observing whether its coordinates are relatively prime as a whole. End of proof.

PROOF OF THEOREM 2.1. We start with the multiple sum

$$
\sum_{a_{1}, a_{2}, \ldots, a_{n} \in z^{*}} \frac{x_{1}^{a_{1}} x_{2}^{a_{2}} \ldots x_{n}^{a_{n}}}{a_{1}^{b_{1}} a_{2}^{b_{2}} \ldots a_{n}^{b_{n}}}=\prod_{t=1}^{n}\left(\sum_{j=1}^{\infty} \frac{x_{1}^{j}}{j^{b_{1}}}\right),
$$

which, due to Lemma 2.3, also equals (letting $b=\sum_{t-1}^{n} b_{\imath}$ )

$$
\begin{array}{r}
\sum_{\substack{\left.a_{1}, a_{2}, \ldots, a_{n}\right)-1 \\
a_{t} \in z^{*}}}\left(\frac{\left(x_{1}^{a_{1}} x_{2}^{a_{2}} \ldots x_{n}^{a_{n}}\right)^{1}}{1^{b}}+\frac{\left(x_{1}^{a_{1}} x_{2}^{a_{2}} \ldots x_{n}^{a_{n}}\right)^{2}}{2^{b}}+\frac{\left(x_{1}^{a_{1}} x_{2}^{a_{2}} \ldots x_{n}^{a_{n}}\right)^{3}}{3^{b}}+\frac{\left(x_{1}^{a_{1}} x_{2}^{a_{2}} \ldots x_{n}^{a_{n}}\right)^{4}}{4^{b}}+\ldots\right) \frac{1}{a_{1}^{b_{1}} a_{2}^{b_{2}} \ldots a_{n}^{b_{n}}} \\
=\sum_{\substack{\left(a_{1}, a_{2}, \ldots, a_{n}\right)-1 \\
a_{1} \in z^{*}}} \frac{-1}{a_{1}^{b_{1}} a_{2}^{b_{2}} \ldots a_{n}^{b_{n}}} \log \left(1-x_{1}^{a_{1} x_{2}^{a_{2}} \ldots x_{n}}\right) \text { if } b=1 .
\end{array}
$$

Exponentiating both sides then yields Theorem 2.1. End of proof.

The cases with $n=2$ and $n=3$ are stated easily, in the forms

$$
\begin{gathered}
\prod_{\substack{(a, b)-1 \\
a, b \in z^{+}}}\left(1-x^{a} y^{b}\right)^{-a^{-s} b^{-t}}=\exp \left\{\left(\sum_{i=1}^{\infty} \frac{x^{t}}{i^{s}}\right)\left(\sum_{j=1}^{\infty} \frac{y^{j}}{j^{t}}\right)\right\}, s+t=1 ; \\
\prod_{\substack{(a, b, c)=1 \\
a, b, c \in z^{*}}}\left(1-x^{a} y^{b} z^{c}\right)^{-a^{--b} b^{-t} c^{-4}}=\exp \left\{\left(\sum_{i=1}^{\infty} \frac{x^{t}}{i^{s}}\right)\left(\sum_{j=1}^{\infty} \frac{y^{j}}{j^{t}}\right)\left(\sum_{k=1}^{\infty} \frac{z^{k}}{k^{u}}\right)\right\}, \quad s+t+u=1 ;
\end{gathered}
$$

where $|x|,|y|,|z|<1$, and $s, t, u$, are complex numbers.

The case of (2.2) with $s=0$ is equivalent to (1.3). In fact (2.2) is a companion to a result given in Campbell [4], namely, for positive integers $m$,

$$
{ }_{k} \Pi_{j}\left(1-x^{j} y^{k}\right)^{j^{m} / k^{m+1}}=\exp \left\{-\left(\sum_{i=1}^{\infty} i^{m} x^{i}\right)\left(\sum_{j=1}^{\infty} \frac{y^{j}}{j^{m+1}}\right)+\sum_{k=0}^{m}\left(\begin{array}{c}
m \\
k
\end{array}\right)\left(\sum_{i=1}^{\infty} i^{k} x^{k}\right)\left(\sum_{j=1}^{\infty} \frac{\left(x y y^{j}\right.}{j^{k+1}}\right)\right\},
$$

where, it was shown in [4], if the power series in $x$ on the right side of (2.4) are replaced by 


$$
g_{m}(x)=\left.\frac{d^{m}}{d z^{m}}\left(1-e^{z}\right)^{-1}\right|_{z-\log x}
$$

then (2.4) holds for $|x y|$ and $|y|<1$.

We shall investigate such companion identities in the next section. As indicated in $\S 1$, such results can imply a large range of new and curious identities, some of which were alluded to in Campbell [5]. However, the classical methods of asymptotics of infinite product generating functions as given in Andrews [1, Chapter 6] will apply to each of the numerous infinite products stated and implied here. Lemma 2.3 is also applicable to many of the lattice sums given in Glasser and Zucker [15] and to the lattice sums presented with applications to mathematical physics as given in Frankel, Glasser, Hughes and Ninham [14]. The latter paper applies simple Möbius and Mellin inversions to lattice sums, obtaining selected applications to electrostatics and statistical mechanics. In the sequel here, we may also draw parallel conclusions to some of the ideas stated in Andrews [1, Chapter 12] describing partitions into vectors. In the present paper we have examples of "weighted" vector partitions, and quite a diversity of results.

The simplicity of our proof of Theorem 2.1 depended largely on the fact that our chosen "raying from origin" region was the first hyperquadrant, that is, the region having all positive integer coordinates in the $n$-space concerned. This meant we had no difficulty in seeing that the multiple summation was simply a product of summations of the same kind on the different variables. For other choices of "rayed from origin" regions this is clearly not such a straightforward matter. It is not, however, an impossible matter. To demonstrate this, we apply Lemma 2.3 to the region in 3-space defined by $1 \leq X<Y<Z$, noting that it rays out of the origin in the manner required. The elements of the set of vectors from the origin to the lattice points in this region can be easily written down, and are countable, as we would expect. Thus we have

$$
\begin{aligned}
& \langle 1,2,3\rangle, \\
& \langle 1,2,4\rangle,\langle 1,3,4\rangle,\langle 2,3,4\rangle, \\
& \langle 1,2,5\rangle,\langle 1,3,5\rangle,\langle 1,4,5\rangle,\langle 2,3,5\rangle,\langle 2,4,5\rangle,\langle 3,4,5\rangle, \\
& \langle 1,2,6\rangle,\langle 1,3,6\rangle,\langle 1,4,6\rangle,\langle 1,5,6\rangle,\langle 2,3,6\rangle,\langle 2,4,6\rangle,\langle 2,5,6\rangle,\langle 3,4,6\rangle,\langle 3,5,6\rangle,\langle 4,5,6\rangle,
\end{aligned}
$$

and so on. A suitable mapping from these vectors results in the summation

$$
\sum_{\substack{0<a<b<c \\ a, b, c \in z^{+}}} x^{a} y^{b} z^{c} c^{-1}
$$

which formally reduces to

$$
\sum_{k=1}^{\infty} \frac{z^{2+k}}{2+k} \frac{x y^{2}}{1-x}\left(\frac{1-y^{k}}{1-y}-x \frac{1-x^{k} y^{k}}{1-x y}\right)
$$

which converges for $|z|,|y z|,|x y z|<1$ to (for non-zero denominators)

$$
\begin{gathered}
\frac{-x y z\left(2 x y-\frac{1}{2} y z+\frac{1}{2} x y z-2\right)}{(1-x)(1-y)(1-x y)}+\frac{x}{(1-x)(1-y)} \log (1-y z)+\frac{1}{(1-x)(1-x y)} \log (1-x y z) \\
-\frac{x y^{2}}{(1-y)(1-x y)} \log (1-z)
\end{gathered}
$$

A similar mapping process to that yielding (2.6) can be applied to the vpv's in the region to obtain the sum

$$
\sum_{\substack{(a, b, c)-1 \\ 0<a<b<c \\ a, b, c \in z^{*}}} x^{a} y^{b} z^{c} c^{-1},
$$


convergent for the same conditions as the sum which led to (2.7). Application of Lemma 2.3 then transforms (2.8) into (2.7), much in the way we proved Theorem 2.1. This leads us to

THEOREM 2.4. If $x, y, x y \neq 1$, and $|z|,|y z|,|x y z|<1$, then

$$
\prod_{\substack{(a, b, c)-1 \\ 0<a<b<c \\ a, b, c \in z^{+}}}\left(1-x^{a} y^{b} z^{c}\right)^{-1 / c}=(1-y z)^{\frac{1}{(1-x)(1-y)}}(1-x y z)^{\frac{1}{(1-x)(1-x y)}}(1-z)^{\frac{-x y^{2}}{(1-y)(1-x y)}} G(x, y, z),
$$

where

$$
G(x, y, z)=\exp \left\{\frac{-x y z\left(2 x y z-\frac{1}{2} y z+\frac{1}{2} x y z-2\right)}{(1-x)(1-y)(1-x y)}\right\} .
$$

Although this theorem looks quite impressive to the reader new to such ideas, it is clearly only comparable to say, a corollary of Theorem 2.1 when apparent depth and dimensionality are considered. The problems faced in trying to make Theorem 2.4 a corollary of Theorem 2.1 involve: firstly, how to divide the hyperquadrant region into subregions, one of which is the region relevant to Theorem 2.4; secondly, to then find the appropriate transform so that not just the right region is covered, but that the index $"-1 / c$ " in the left side product is obtainable. In many cases it seems more practical to choose a region and apply Lemma 2.3 , but there will be situations worth examining where the hyperspace divides naturally into regions yielding companion identities. In order to classify all such identities it may be necessary to use group theory and isometries.

So it seems that (2.9) is not in any obvious sense a companion identity for (2.3), nor for a case of it. Similarly, this appears so for the following result, obtained from summing on the lattice points in the inverted right square pyramid $|X|$ and $|Y|<Z$ in the $X-Y-Z$ space.

THEOREM 2.4. If $x, y, 1-x, 1-y$, are all non-zero, and each of $|z|,|x z|,|z / x|,|y z|,|z / y|$, $|x y z|,|z /(x y)|,|x z / y|,|y z / x|<1$, then

$$
\prod_{\substack{a, b, c)-1 \\|a|,|b| c \in z^{+} \\ a, b \in z}}\left(1-x^{a} y^{b} z^{c}\right)^{1 / c}=\left(\frac{(1-z)(1-x y z)^{x y}}{(1-x z)^{x}(1-y z)^{y}}\right)^{\frac{1}{x y(1-x)(1-y)}}
$$

This result suggests the existence of an analogue of Theorem 2.1 where summation is taken over the vpv's and lattice points in the appropriately defined hyperpyramid in Euclidean $\boldsymbol{n}$-space. Clearly the hyperquadrant appears not to easily transform into the hyperpyramid, and vice versa, thus a totally different set of companion identities are implied. There are also problems related to the consideration of the branching effects of the complex roots involved. For example, in exactly which sense do both sides of (2.10) satisfy the functional equation $f(x, y, z)=f(1 / x, 1 / y, 1 / z)$ ?

\section{NATURAL CASES AND A FURTHER $\boldsymbol{N}$-SPACE THEOREM}

There are natural and simple cases of Theorem 2.1 to consider. Let us first enlarge the theorem's positive coordinate hyperquadrant to include lattice points on each axis except for the highest or $n$th dimension. Thus we can easily obtain the following

COROLLARY 3.1.

$$
\begin{aligned}
& \prod_{\substack{(a, b)=1 \\
a \geq 0, b \geq 1}}\left(1-x^{a} y^{b}\right)^{1 / b}=(1-y)^{1 /(1-x)}, \\
& \prod_{\substack{(a, b, c)-1 \\
a, b \geq 0, c \geq 1}}\left(1-x^{a} y^{b} z^{c}\right)^{1 / c}=(1-z)^{1 /(1-x)(1-y))},
\end{aligned}
$$




$$
\begin{gathered}
\prod_{\substack{(a, b, c, d)-1 \\
a, b<\geq 0, d<1}}\left(1-v^{a} x^{b} y^{c} z^{d}\right)^{1 / d}=(1-z)^{1 /(1-v)(1-x)(1-y)),} \\
\prod_{\substack{(a b, c, d, c)-1 \\
a, b, c, d \geq 0, c \geq 1}}\left(1-u^{a} v^{b} x^{c} y^{d} z^{c}\right)^{1 / c}=(1-z)^{1 /(1-u)(1-v)(1-x)(1-y)),}
\end{gathered}
$$

each valid when absolute values of $u, v, x, y$, and $z$ are all less than unity.

The $n$-space version is then

COROLLARY 3.2. If for $i=1,2, \ldots, n ;\left|x_{i}\right|<1$ and $|z|<1$, then

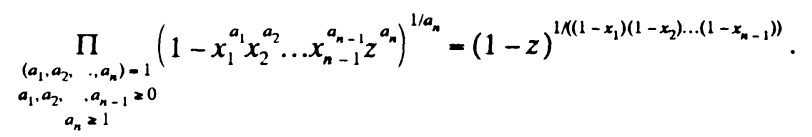

At this point we can apply Lemma 2.3 to Corollary 3.2. In other words we get rid of the criterion on the left side product from $(3.5)$ that $\left(a_{1}, a_{2}, \ldots, a_{n}\right)=1$. We shall rate the result of this as a theorem since the subsequent corollaries will be of a distinctly different kind from our earlier identities.

THEOREM 3.3. For the same conditions as (3.5) we have

$$
\prod_{\substack{a_{1}, a_{2}, \ldots, a_{n-1} \geq 0 \\ a_{n} \geq 1}}\left(1-x_{1}^{a_{1}} x_{2}^{a_{2}} \ldots x_{n-1}^{a_{n-1}} z^{a_{n}}\right)^{1 / a_{n}}=\prod_{\lambda=1}\left(1-z^{\lambda}\right)^{\frac{(1 / \lambda)}{\left(1-x_{1}^{\lambda}\right)\left(1-x_{2}^{\lambda}\right) \ldots\left(1-x_{n-1}^{\lambda}\right)}} .
$$

PROOF. If the left side of $(3.5)$ is defined by $f\left(x_{1}, x_{2}, \ldots, x_{n-1}, z\right)$ then by Lemma 2.3 this transforms into the left side of (3.6) by forming

$$
\prod_{\lambda=1} f\left(x_{1}^{\lambda}, x_{2}^{\lambda}, \ldots, x_{n-1}^{\lambda}, z^{\lambda}\right)^{1 / \lambda} .
$$

Applying the same operation to the right side of (3.5) yields the right side of (3.6). End of proof.

We can allow $n \rightarrow \infty$ in Theorem 3.3 to obtain some interesting results. Firstly, if $x_{i}-p_{i}^{-s}$ with $p_{i}$ denoting the $i$ th prime we have

COROLLARY 3.4. If $\operatorname{Re} s>1$ and $|z|<1$

$$
\prod_{j, k \geq 1}\left(1-\frac{z^{2 k}}{j^{s}}\right)^{1 / k}=\prod_{\lambda \geq 1}\left(1-x^{2 \lambda}\right)^{(\lambda \lambda s) \lambda}
$$

Particular cases of this lead to the further

COROLLARY 3.5. If $|z|<1$

$$
\begin{gathered}
\prod_{k \geq 1}\left(\frac{\sin \left(\pi z^{k}\right)}{\pi z^{k}}\right)^{1 / k}=\prod_{\lambda \geq 1}\left(1-x^{2 \lambda}\right)^{\xi(2 \lambda) / \lambda}, \\
\prod_{k \geq 1}\left(\cos \left(\pi z^{k}\right)\right)^{1 / k}=\prod_{\lambda \geq 1}\left(1-z^{2 \lambda}\right)^{3 \xi(2 \lambda) / 4 \lambda}, \\
\prod_{k \geq 1}\left(\frac{\sinh \left(2 \pi z^{k}\right)-2 \sinh \left(\pi z^{k}\right) \cos \left(\pi z^{k} \sqrt{3}\right)}{4 \pi^{3} z^{3 k}}\right)^{1 / k}=\prod_{\lambda \geq 1}\left(1+z^{6 \lambda}\right)^{\xi(6 \lambda) / \lambda}
\end{gathered}
$$

The latter of these results is affected with the aid of the following product apparently due to Ramanujan. If $x$ is any non-zero complex number

$$
\prod_{n=1}^{\infty}\left(1+\frac{x^{6}}{n^{6}}\right)=\frac{\sinh (2 \pi x)-2 \sinh (\pi x) \cos (\pi x \sqrt{3})}{4 \pi^{3} x^{3}}
$$

For a proof of this see Berndt [3, pp. 107-108]. 
Again employing the case of Theorem 3.3 with $n \rightarrow \infty$ and this time letting $x_{t}=x^{\prime}$ we can obtain new relations involving $p(n)$, the number of unrestricted partitions of $n$. It is well known that (see Andrews [1])

$$
\sum_{n=0}^{\infty} p(n) x^{n}=\prod_{k=1}^{\infty}\left(1-x^{k}\right)^{-1}:=P(x), \quad|x|<1 .
$$

Likewise, if $p_{o}(n)$ is the number of partitions of $n$ into odd parts

$$
\sum_{n=0}^{\infty} p_{o}(n) x^{n}=\prod_{k-1}^{\infty}\left(1-x^{2 k-1}\right)^{-1}:=P_{o}(x),|x|<1 .
$$

COROLLARY 3.6. If $|x|$ and $|z|<1$

$$
\begin{aligned}
& \prod_{\substack{j \geq 0 \\
k \geq 1}}\left(1-x^{j} z^{k}\right)^{p(y) k}=\prod_{\lambda \geq 1}\left(1-x^{\lambda}\right)^{\left.\mathcal{F} x^{\lambda}\right) \lambda,} \\
& \prod_{\substack{j \geq 0 \\
k \geq 1}}\left(1-x^{\prime} z^{k}\right)^{p_{0}(1) k}=\prod_{\lambda \geq 1}\left(1-x^{\lambda}\right)^{p_{0}\left(x^{\lambda}\right) / \lambda},
\end{aligned}
$$

Clearly there is scope for further research into identities such as these, especially given the already existing wealth of material on partition identities. An alternative version of (3.13) for the same conditions is

$$
\prod_{,, k=1}\left(1-x^{J} z^{k}\right)^{p(y) k}=\prod_{k=1} \prod_{d \mid k}\left(1-x^{d}\right)^{(p(k) d) d) x^{k}} .
$$

Another natural concept arising from the above work is $p_{\ell}(j)$, the number of partitions of $j$ into parts at least one of which is relatively prime to $k$. In other words $\left(a_{1}, a_{2}, \ldots, a_{m}, k\right)=1$, or equivalently an $a_{i} \in \phi_{k}$ from $\S 1$ and the form taken for such a partition of $j$ is then

$$
j=A_{1} a_{1}+A_{2} a_{2}+\ldots+A_{m} a_{m}, \quad \text { (for any } m \geq 1 \text { ) }
$$

where the $A_{i}$ are positive integers without restriction. Setting $\mathrm{p}_{k}(0):=1$ and employing our definition, then allowing $n \rightarrow \infty$ in Corollary 3.2 setting $x_{i}=x^{i}$, the result follows that

COROLLARY 3.7. If $|x|$ and $|z|<1$ then for $\mathcal{P}(x)$ as in (3.11),

$$
\prod_{\substack{j \geq 0 \\ k \geq 1}}\left(1-x^{j} z^{k}\right)^{P_{k}(U) / k}=(1-z)^{f(x)} \text {. }
$$

\section{COMPANION IDENTITIES AND VISIBLE POINT VECTOR PARTITIONS}

In the book on Partitions by G. E. Andrews [1, Chapter 12] it is stated that "we do not have a device like Euler's pentagonal number theorem for the rapid calculation of any multipartite functions." The preceding sections of this paper and its predecessors [4 to 6] indicate developments in this direction, due to the often easy expansion of the functions concerned. e.g. The right sides of (3.2) through to (3.6) are easily expanded as power series in $z$. Andrews mentions how Carlitz and others [7 to 9, and 11,12] considered problems of restricted multipartite partitions. In particular, $k$-partite partitions

$$
\left\langle n_{1}, n_{2}, \ldots, n_{k}\right\rangle=\sum_{j=1}^{r}\left\langle m_{i j}, m_{2 j}, \ldots, m_{k j}\right\rangle
$$

subject to the decreasing condition

$$
\min \left(m_{t \jmath}, m_{2 j}, \ldots, m_{k j}\right) \geq \max \left(m_{1, j+1}, m_{2, j+1}, \ldots, m_{k, j+1}\right) .
$$

It is shown in [1] that if $\Pi\langle n, m\rangle$ is the number of partitions of $\langle n, m\rangle$ into steadily decreasing parts then for $|x|$ and $|y|<1$ 


$$
\sum_{n, m \geq 0} \Pi\langle n, m\rangle x^{n} y^{m}=\prod_{n=1}\left(1-x^{n} y^{n-1}\right)^{-1}\left(1-x^{n-1} y^{n}\right)^{-1}\left(1-x^{2 n} y^{2 n}\right)^{-1} .
$$

From this it follows easily that if $\Pi_{1}\langle n, m\rangle$ is the number of bipartite partitions of $\langle n, m\rangle$ in which all parts are of the forms $\langle 2 a, 2 a\rangle$ or $\langle a-1, a\rangle$ or $\langle a, a-1\rangle$ then for all $n$ and $m, \Pi\langle n, m\rangle=\Pi_{1}\langle n, m\rangle$. Andrews states that this result is of a different kind than those arising from the simple extensions of partition ideals of order 1 considered in his book.

In the light of our work in this paper it now becomes natural to consider partitions into visible point vectors (vpv's) within rayed-from-origin regions of vector spaces. Our notion of "companion identities," imprecise though it be at this state, due to ambiguities as presented, will still be useful when we obtain theorems involving "the number of vpv partitions" of various kinds of vectors.

As promised in $\S 1$, we now present a generalization of the companion identities (1.1), (1.2), and (1.3).

CONJECTURE 4.1. Let $s$ and $t$ be complex and such that $s+t=1$. Then respectively for $|x y|$ and $|y|<1,|x|$ and $|x y|<1,|x|$ and $|y|<1$,

$$
\begin{aligned}
& { }_{b} \Pi_{a}\left(1-x^{a} y^{b}\right)^{-a^{-s} b^{-1}}=\exp \left\{\left(\sum_{i=1}^{\infty} \frac{x^{\prime}}{i^{s}}\right)\left(\sum_{i=1}^{\infty} \frac{y^{\prime}}{j^{t}}\right)-\sum_{i, j=1}\left(1+\frac{i}{j}\right)^{-s} \frac{x^{\prime+j} y^{\prime}}{j}\right\}, \\
& (1-x y)_{b} \Pi_{a}\left(1-x^{b} y^{a}\right)^{-b^{-s} a^{-t}}=\exp \left\{-\sum_{i, j \geq 1}\left(1+\frac{i}{j}\right)^{-s} \frac{x^{i+j} y^{j}}{j}\right\} \text {, } \\
& (1-x y)_{b} \Pi_{a}\left(1-x^{a} y^{b}\right)^{-a^{-s} b^{-1}}\left(1-x^{b} y^{a}\right)^{b^{-s} a^{-1}}=\exp \left\{\left(\sum_{1}^{\infty} \frac{x^{\prime}}{i^{s}}\right)\left(\sum_{-1}^{\infty} \frac{y^{\prime}}{j^{\prime}}\right)\right\},
\end{aligned}
$$

where it is understood that any divergent multilogarithmic power series on the right sides can be replaced by the function defined in (2.5).

Partial proof. (4.3) is seen to be equivalent to (2.2). Similarly, (2.4) is the case of (4.1) where for positive integers $m, t=m+1$. However, (4.1) claims considerably more than (2.4); namely, that $s$ and $t$ can be complex numbers. (4.3) is obviously true for such $s$ and $t$, so it would appear natural for (4.1) to fulfill these conditions. Since (4.2) is a simple quotient of both sides of (4.3) over (4.1) it remains only for us to establish that (4.1) is true for complex $s$ and $t$. In Campbell [4] the proof of (2.4) depended on an $m$ th derivative with respect to $z=\log x$ of

$$
f(n, y)+{ }_{k} \sum_{j} f\left(n, x^{\prime} y^{k}\right) k^{-n}=(f(n, y)-f(n, x y)) /(1-x),
$$

where $n \in \mathbf{C}$ and $f(n, y)=\sum_{k=1}^{\infty} y^{k} n^{-n}$ with $|y|<1$. This led to

$$
{ }_{k} \sum,(j / k)^{m} f\left(n, x^{j} y^{k} k\right) k^{-n}=f(n+m, y) g_{m}(x)-\sum_{k=0}^{\infty}\left(\begin{array}{c}
m \\
k
\end{array}\right) f(n+k, x y) g_{k+1}(x),
$$

for non-negative integers $m$ and $g_{m}(x)$ as in (2.5). Allowing $n=1$ in this yields (2.4) and hence (4.1) and (4.2) for the values $t=m+1 x$. To fully prove conjecture 4.1 would require complex iteration on the derivative process applied above to (4.4). End of partial proof.

It seems worth noting at this stage, having just failed to prove conjecture 4.1 completely, that under the conditions specified there, Lemma 2.3 when applied directly gives

$$
\begin{gathered}
{ }_{b} \Pi_{a}\left(1-x^{a} y^{b}\right)^{-a^{-s} b^{-t}}=\exp \left(\frac{x y^{2}}{1^{s} 2^{t}}+\left(\frac{x}{1^{s}}+\frac{x^{2}}{2^{s}}\right) \frac{y^{3}}{3^{t}}+\left(\frac{x}{1^{s}}+\frac{x^{2}}{2^{s}}+\frac{x^{3}}{3^{s}}\right) \frac{y^{4}}{4^{t}}+\ldots\right), \\
(1-x y)_{b} \Pi_{a}\left(1-x^{b} y^{a}\right)^{-b^{-s} a^{-t}}=\exp \left(x y+\left(\frac{y}{1^{s}}+\frac{y^{2}}{2^{s}}\right) \frac{x^{2}}{2^{t}}+\left(\frac{y}{1^{s}}+\frac{y^{2}}{2^{s}}+\frac{y^{3}}{3^{s}}\right) \frac{x^{3}}{3^{t}}+\left(\frac{y}{1^{s}}+\frac{y^{2}}{2^{s}}+\frac{y^{3}}{3^{s}}\right) \frac{x^{3}}{3^{t}}+\ldots\right),
\end{gathered}
$$


and of course, (4.3). This appears to add further weight to the likelihood of conjecture 4.1 being true. It also shows that the summations exponentiated on the right sides of (4.6) and (4.7) are each valuable in terms of a finite sum of multilogarithms (or polylogarithms, see Lewin [16], and [17]) whenever $t=m+1$ for non-negative integer $m$, with $s+t=1$. This could no doubt be developed further in a later paper.

At this stage, let us return to the idea of vector partitions. More specifically, we consider vpv's in the first quadrant of the $X-Y$ plane. Basically, the vpv's can be divided into three classes:

a) those above the line $X=Y$, the upper vpv's,

b) those below and on the line $X=Y$, the lower vpv's,

c) those of the entire quadrant, that is, all vpv's.

We shall therefore look at $\mathbf{U}\langle\mathbf{n}, \mathbf{m}\rangle, \mathbf{L}\langle\mathbf{n}, \mathbf{m}\rangle, \mathbf{A}\langle\mathbf{n}, \mathbf{m}\rangle$; the number of partitions of $\langle n, m\rangle$ into respectively, upper, lower, and all vpv's. Using the techniques in Andrews [1], it is a simple matter to show that

$$
\begin{gathered}
1+\sum_{1 \leq n<m} \mathbf{U}\langle\mathbf{n}, \mathbf{m}\rangle x^{n} y^{m}={ }_{k} \Pi_{j}\left(1-x^{j} y^{k}\right)^{-1}, \\
1+\sum_{1<+m<n} \mathbf{L}\langle\mathbf{n}, \mathbf{m}\rangle x^{n} y^{m}=(1-x y)_{k}^{-1} \Pi_{j}\left(1-x^{k} y^{j}\right)^{-1}, \\
1+\sum_{n, m=1} \mathbf{A}\langle\mathbf{n}, \mathbf{m}\rangle x^{n} y^{m}=(1-x y)^{-1} \Pi_{k}\left(1-x^{j} y^{k}\right)^{-1}\left(1-x^{k} y^{j}\right)^{-1},
\end{gathered}
$$

valid for the same respective conditions on $x$ and $y$ as imposed on (1.1), (1.2) and (1.3).

Each side of (4.8) satisfies the functional equation

$$
f(x, y)=f(1 / x, x y),
$$

provided $f(1 / x, y)$ is first taken before proceedings to $f(1 / x, x y)$, the operations being not commutative. The coefficients of $y^{m}$ in (4.8) are the polynomials

$$
\sum_{n=1}^{m-1} \mathbf{U}\langle\mathbf{n}, \mathbf{m}\rangle x^{n}
$$

It therefore follows naively but significantly that,

THEOREM 4.2. For all positive integers $n, m, \mathbf{U}\langle\mathbf{m}-\mathbf{n}, \mathbf{m}\rangle=\mathbf{U}\langle\mathbf{n}, \mathbf{m}\rangle$.

The reasoning behind this theorem can equally be applied to numerous other results involving vpv identities. For example, (4.9) and (1.2) both satisfy the functional equation

$$
f(x, y)=\frac{1-x}{1-x y} f(x y, 1 / y),
$$

where in this case $f(x, 1 / y)$ precedes arrival at $f(x y, 1 / y)$. For this reason we formalize the following

DEFINITION 4.3. Let $\rho$ be a mapping applied to $U\langle n, m\rangle, L\langle n, m\rangle, A\langle n, m\rangle$, respectively to yield $\rho_{u}\langle\mathbf{n}, \mathbf{m}\rangle, \rho_{l}\langle\mathbf{n}, \mathbf{m}\rangle, \rho_{\mathbf{a}}\langle\mathbf{n}, \mathbf{m}\rangle$. If

$$
\begin{aligned}
& 1+\sum_{1<+n<m}\left\langle\rho_{\mathrm{u}}, \mathrm{m}\right\rangle x^{n} y^{m}=F_{\mathrm{U}}(x, y), \\
& 1+\sum_{1<+n<m}\left\langle\rho_{l}, \mathrm{~m}\right\rangle x^{n} y^{m}=F_{\mathrm{L}}(x, y), \\
& 1+\sum_{n, m \geq 1}\left\langle\rho_{\mathrm{u}}, \mathrm{m}\right\rangle x^{n} y^{m}=F_{\mathrm{A}}(x, y),
\end{aligned}
$$

then $F_{\mathrm{u}}(x, y), F_{\mathrm{L}}(x, y), F_{\mathrm{A}}(x, y)$, are respectively called "upper vpv", "lower vpv", "all vpv" functions; and likewise (4.14), (4.15), (4.16), called "upper vpv", "lower vpv", "all vpv" identities.

This definition will allow us to consider the two dimensional identities in more detail, and to be more specific when analyzing them and related results. We mentioned in $\$ 1$ how each of the "companions" (1.1), (1.2), (1.3) implies each the other two. The following three statements can be equivalent: 


$$
\begin{gathered}
F_{\mathrm{A}}(x, x y)=F_{\mathrm{L}}(x, y), \\
F_{\mathrm{A}}(x, y)=\sum_{k=0}^{\infty} F_{\mathrm{U}}\left(x, x^{k} y\right), \\
F_{\mathrm{U}}(x, y) F_{\mathrm{L}}(x, y)=F_{\mathrm{A}}(x, y) .
\end{gathered}
$$

In other words, if given a suitable "upper", "lower", or "all" vpv identity, we can manufacture a set of companion identities. (1.1), (1.2) and (1.3) are essentially an example of this. Rather than labor this point, we simply present the "all vpv" identity resulting from applying (4.18) to (2.4).

THEOREM 4.4. If $|x|$ and $|y|<1, g_{h}(x)$ as in (2.5), and $h$ is any positive integer, then

$$
\prod_{\substack{a, b)-1 \\
a, b \in z^{*} \\
a=d(\bmod b)}}\left(1-x^{a} y^{b}\right)^{d^{h} / b^{h+1}}=(1-x y) \exp \left(-g_{h}(x) \sum_{j=1}^{\infty} \frac{y^{j}}{j^{h+1}\left(1-x^{j}\right)}+\sum_{k=0}^{\infty}\left(\begin{array}{l}
h \\
k
\end{array}\right) g_{k}(x) \sum_{j=1}^{\infty} \frac{x^{j} y^{j}}{j^{k+1}\left(1-x^{j}\right)}\right) \text {. }
$$

This theorem is an almost unrecognizable generalization of (1.3), which would correspond to the case $\boldsymbol{h}=0$. It is distinct from the other types of "all vpv" identities given so far. The cases with $\boldsymbol{h}=1$ and $h=2$ are, with $f(n, y)$ as in (4.4),

$$
\prod_{\substack{(a, b)-1 \\ a, b \in \in z^{*} \\ a \in d(\bmod b)}}\left(1-x^{a} y^{b}\right)^{d^{1} / b^{2}}=\exp \left\{x f(2, y)(1-x)^{-2}\right\} \prod_{k=1}^{\infty}\left(1-x^{k} y\right)^{-1 / 1-x)},
$$

$$
\prod_{\substack{(a, b)-1 \\ a, b \in z^{*} \\ a=d(\bmod b)}}\left(1-x^{a} y^{b}\right)^{d^{2} / b^{3}}=\exp \left\{-\left(x+x^{2}\right)(1-x)^{-3} f(3, y)-x(1-x)^{-2} f(2, y)\right\} \prod_{k=1}^{\infty}\left(1-x^{k} y\right)^{-1 /(1-x)}
$$

Yet another method (and perhaps the most obvious) for manufacturing related "companions" involves simply the two equations

$$
F_{\mathrm{u}}(x, y)=F_{\mathrm{A}}(x y, y) \text {, and } F_{\mathrm{L}}(x, y)=F_{\mathrm{A}}(x, x y) \text {. }
$$

When using this method an adjustment may be made to the first term of an identity to tidy things up. Applying (4.23) to the "all vpv" (2.2) gives the "upper" and "lower" vpv identities, even though they are trivially interdependent.

COROLLARY 4.5. If $s$ and $t$ are complex and $s+t=1$, whilst $|x y|$ and $|y|<1$ for (4.24); $|x|$ and $|x y|<1$ for (4.25), then

$$
\begin{aligned}
& { }_{k} \Pi_{j}\left(1-x^{j} y^{k}\right)^{-j^{-s}(k-j)^{-1}}=\exp \left\{\left(\sum_{i=1}^{\infty} \frac{(x y)^{i}}{i^{s}}\right)\left(\sum_{j=1}^{\infty} \frac{y^{j}}{j^{j}}\right)\right\}, \\
& { }_{k} \Pi_{j}\left(1-x^{k} y^{j}\right)^{-(k-j)^{-j} j^{-1}}=\exp \left\{\left(\sum_{i=1}^{\infty} \frac{x^{i}}{i^{s}}\right)\left(\sum_{j=1}^{\infty} \frac{(x y)^{j}}{j^{t}}\right)\right\} .
\end{aligned}
$$

This corollary highlights the possibilities for writing down different classes of companion identities, each set of "companions" having often strikingly different appearance, even when two distinct companion sets may have one identity shared, as was the case in this section. We mention at this juncture, that nearly all of the companions given so far have corollary companions derived from replacing certain of the variables by their squares, then from the resulting identities, dividing through by the original identity. To illustrate such results we give as an example from (1.1), (1.2), and (1.3), 
COROLLARY 4.6. For respective conditions on $x$ and $y$ as in (1.1), (1.2), and (1.3) we have

$$
\begin{gathered}
(1+y)_{k} \Pi_{j}\left(1+x^{j} y^{k}\right)^{1 / k}=\left(\frac{(1-x y)^{x}(1+y)}{(1-y)^{k}(1+x y)}\right)^{1 /\left(1-x^{2}\right)}, \quad x \neq 1, \\
(1+x y)_{k} \Pi_{j}\left(1+x^{k} y^{\prime}\right)^{1 / j}=\left(\frac{1+x y}{(1-x y)^{x}}\right)^{1 /\left(1-x^{2}\right)}, \\
(1+y)(1+x y)_{k} \Pi_{j}\left(1+x^{\prime} y^{k}\right)^{1 / k}\left(1+x^{k} y^{j}\right)^{1 / j}=\left(\frac{1+y}{(1-y)^{x}}\right)^{\left.1 / 1-x^{2}\right)} .
\end{gathered}
$$

Application of the same method to (2.10) yields:

COROLLARY 4.7. For $x, y$, and $z$ as in (2.10),

$$
\prod_{\substack{a, b, c)-1 \\|a|,|b|<c \in z^{+} \\ a, b \in z}}\left(1+x^{a} y^{b} z^{c}\right)^{1 / c}=\left(\frac{(1+z)^{\mathrm{a}}(1+x y z)^{\mathrm{b}}(1-x)^{\mathrm{c}}(1-y z)^{\mathrm{d}}}{(1-z)^{\mathrm{e}}(1-x y z)^{\mathrm{d}}(1+x z)^{\mathrm{g}}(1+y z)^{\mathrm{h}}}\right)^{\frac{1}{x^{2} y^{2}\left(1-x^{2}\right)\left(1-y^{2}\right)}},
$$

where $\mathrm{a}=1, \mathrm{~b}=x^{2} y^{2}, \mathrm{c}=x^{2} y(1+x)(1+y)-x^{2}, \mathbf{d}=y^{2} x(1+x)(1+y)-y^{2}, \mathrm{e}=x y(1+x)(1+y)-1$, $\mathbf{f}=x^{2} y^{2}(1+x)(1+y)-x^{2} y^{2}, \mathbf{g}=x^{2}, \mathbf{h}=y^{2}$.

It should be clear to the reader that (4.29) can, like (2.10), be derived from first principles by taking an alternating sum on the lattice points and vpv's in the rayed-from-origin region bounded by the inverted right square defined in $X-Y-Z$ space by $|X|$ and $|Y|<Z$.

In Campbell [4], similar suggestions for obtaining corollaries were given. Another neat corollary is obtained by swapping $s$ and $t$ in (4.25), then dividing both sides of this into (4.24), so then

COROLLARY 4.8. If $s$ and $t$ are complex with $s+t=1,|x|$ and $|y|<1$,

$$
{ }_{k} \Pi_{j}\left(\frac{1-x^{j} y^{k}}{1-x^{k} y^{j}}\right)^{j^{-s}(k-j)^{-t}}=\exp \left(\left(\sum_{j=1}^{\infty} \frac{(x y)^{j}}{j^{s}}\right)\left(\sum_{k=1}^{\infty} \frac{x^{k}-y^{k}}{k^{t}}\right)\right) .
$$

The identities of this section show that many of the simple properties of the companion identities appear to be worth formalizing. For example, (4.30) indicates that the quotient of an "upper vpv" with a "lower vpv" identity yields (or is expected to yield) an "all vpv" identity.

We continue this section by giving a set of companion identities in three dimensional space. In this instance, we consider the first hyperquadrant of the $X-Y-Z$ plane including $X$ and $Y$ axes whilst excluding the $Z$ axis. In fact, if the first hyperquadrant is treated as an infinitely extended cube, then the identities here correspond to the lattice points in:

a) the extended tetrahedron bounded by $X, Y<Z$,

b) the extended tetrahedron bounded by $X, Z \leq Y$,

c) the extended tetrahedron bounded by $Y, Z \leq X$,

d) the entire hyperquadrant which is the union of $a), b$ ), and $c$ ).

THEOREM 4.9. If $a$ and $b$ each sum over the non-negative integers and $c$ the positive integers, such that for $a, b>0,(a, b, c)=1$ then

$$
\begin{gathered}
\prod_{a, b<c}\left(1-x^{a} y^{b} z^{c}\right)^{-1 / c}=\left(\frac{(1-x z)(1-y z)}{(1-z)(1-x y z)}\right)^{1 /(1-x)(1-y)),}, \\
\prod_{a, c \leq b}\left(1-x^{a} y^{b} z^{c}\right)^{-1 / c}=\left(\frac{(1-x y z)^{x y}}{(1-y z)^{y}}\right)^{1 /(1-x)(1-y)),}, \\
\prod_{b, c \leq a}\left(1-x^{a} y^{b} z^{c}\right)^{-1 / c}=\left(\frac{(1-x y z)^{x y}}{(1-x z)^{x}}\right)^{1 /(1-x)(1-y))},
\end{gathered} .
$$




$$
\prod_{a, b, c}\left(1-x^{a} y^{b} z^{c}\right)^{-1 / c}=(1-z)^{-1 /(1-x)(1-y))},
$$

where the conditions for convergence are respectively,

$$
\begin{gathered}
|z|,|x z|,|y z|,|x y z|<1 ; \\
|y z|,|x y z|<1 ; \\
|x z|,|x y z|<1 ; \\
|x|,|y|,|z|<1 ;
\end{gathered}
$$

with variables chosen for non-zero denominators.

PROOF. In the usual manner we consider the four lattice sums:

$$
\sum_{a, b<c} x^{a} y^{b} z^{c} / c, \sum_{a, c=b} x^{a} y^{b} z^{c} / c, \sum_{b, c=a} x^{a} y^{b} z^{c} / c, \sum_{a, b, c} x^{a} y^{b} z^{c} / c
$$

The last of these we have already given in (3.2) as a case of (2.3) basically. The second and third of these are essentially the same as each other, by interchange of $x$ and $y$. They easily reduce to the logarithm of the right sides of (4.32) and (4.33) by use of the summation $(|u|$ and $|z|<1)$

$$
\sum_{j=1}^{\infty}\left(\sum_{k=1}^{j} \frac{z^{k}}{k}\right) u^{j}=\frac{-1}{1-u} \log (1-u z),
$$

as part of the process. The first lattice sum is

$$
\sum_{k=1}^{\infty} \frac{z^{k}}{k} \frac{\left(1-x^{k}\right)\left(1-y^{k}\right)}{(1-x)(1-y)}=\frac{1}{(1-x)(1-y)} \log \frac{(1-x z)(1-y z)}{(1-z)(1-x y z)}
$$

which is the logarithm of the right side of (4.31). Now each of the four lattice sums considered correspond to the aforementioned "infinitely extended" tetrahedra which fit together to make up the first hyperquadrant. As such, each tetrahedron is a "rayed from origin" region as pertains to Lemma 2.3. Therefore, applying Lemma 2.3 to our four lattice sums, we have for example used the first sum that it transforms into

$$
\sum_{k=1}^{\infty} \sum_{(a, b, c)-1}\left(x^{a} y^{b} z^{c}\right)^{k} /(c k)=\sum_{(a, b, c)-1}(-1 / c) \log \left(1-x^{a} y^{b} z^{c}\right),
$$

and this establishes (4.31). Similar reasoning applied to the other three lattice sums gives (4.32), (4.33) and (4.34). End of proof.

There is an interesting resemblance between Theorems 4.9 and 2.4. The sum (4.35) shows that identities like (4.31) can easily be generalized to $n$-dimensional space. Using sums like (4.35) the next few cases are

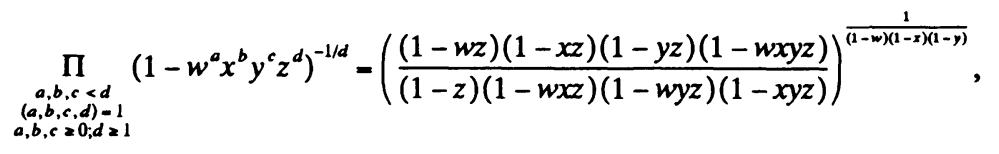

$$
\begin{aligned}
& \prod_{\substack{a, b, c, d<e \\
a, b, c, d, e)-1}}\left(1-v^{a} w^{b} x^{c} y^{d} z^{e}\right)^{-1 / e}- \\
& (a, b, c, d, c)=1 \\
& a, b, c, d \geq 0 ; e \geq 1 \\
& \left(\frac{(1-v z)(1-w z)(1-x z)(1-y z)(1-v w x z)(1-v w y z)(1-v x y z)(1-w x y z)}{(1-z)(1-v w z)(1-v x z)(1-v y z)(1-w x z)(1-w y z)(1-x y z)(1-v w x y z)}\right)^{G(v, w, x, y)},
\end{aligned}
$$

where $G(v, w, x, y)=((1-v)(1-w)(1-x)(1-y))^{-1} ;(4.36)$ and $(4.37)$ need the absolute values of each of $z, v z, w z, x z, \ldots, v w z, \ldots, v w x z, \ldots, v w x y z$ to be appropriately less than unity.

In general dimension we have the following 
THEOREM 4.10. If $\mathbf{X}_{0}\left(x_{1}, x_{2}, \ldots, x_{n}\right)$ and $X_{e}\left(x_{1}, x_{2}, \ldots, x_{n}\right)$ are the sets of respectively odd and even number of products of dissimilar $x_{1}, \ldots, x_{n}$ then for $i=1,2, \ldots, n$ we have (assuming $1=\mathbf{X}_{\mathbf{e}}$ acting on the empty set)

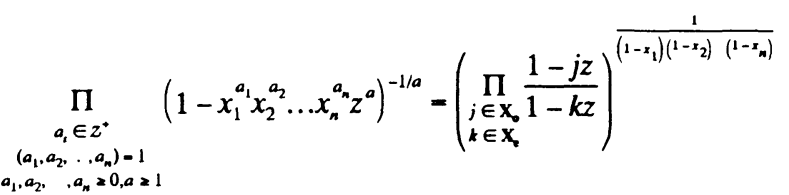

valid for absolute values of "each element product of $\mathbf{X}_{0}$ and $\mathbf{X}_{\mathbf{e}}$ multiplied by $z^{\prime \prime}$ to be less than unity, and also $|z|<1$. Also none of the $x_{1}$ are unity.

This theorem has an interesting connection to partition theory if we permit $n$ to increase indefinitely. After this, letting $x_{i}=x^{1}, p_{1}(0)=1, p_{k}(0)=0(k>1)$

$$
\prod_{, \geq 0, k=1}\left(1-x^{\prime} z^{k}\right)^{-p_{k}(0) / k}=\left(\frac{(1-x z)^{p_{0}(1)}\left(1-x^{2} z\right)^{p_{0}(2)} \ldots}{(1-z)(1-x z)^{p_{0}(1)}\left(1-x^{2} z\right)^{p_{e}(2)} \ldots}\right)^{\frac{1}{\left(1-x^{1}\right)\left(1-x^{2}\right)\left(1-x^{3}\right) .}},
$$

where $p_{k}(j)$ is the number of partitions into parts $<k$, at least one part $\in \phi_{k}$ from $\S 1$,

$p_{0}(j)$ is the number of partitions of $j$ into an odd number of distinct parts,

$p_{e}(j)$ is the number of partitions of $j$ into an even number of distinct parts.

However, Euler's pentagonal number theorem (see Andrews [1, pp. 10]) states that

$$
p_{e}(j)-p_{o}(j)= \begin{cases}(-1)^{m} & \text { if } j=\frac{1}{2} m(3 m \pm 1), \\ 0 & \text { otherwise }\end{cases}
$$

Applying this to the right side of (4.39) gives

COROLLARY 4.11. If $|x|$ and $|z|<1$,

$$
\prod_{j \geq 0, k \geq 1}\left(1-x^{j} z^{k}\right)^{-p_{k}(j) / k}=\left(\frac{1}{1-z} \prod_{j=1}^{\infty}\left(1-x^{\frac{1}{2} j(3 j \pm 1)} z\right)^{(-1)^{\prime+1}}\right)^{\frac{1}{(1-x)\left(1-x^{2}\right)\left(1-x^{3}\right) \ldots}} .
$$

It appears that the results of this section are not the final word on the topic of companion identities and vpv partitions. The connection with the known theory of partitions highlighted by results such as the latter corollary also indicates a rich potential for further development.

\section{EULER PRODUCT IDENTITIES AND MULTIPLICATIVE FUNCTIONS}

The method outlined in $\$ 1$ for obtaining (1.4), (1.5), and (1.6) is, as surmised from Campbell [6], capable of applying to almost all of the identities in our subsequent sections. Basically here we revisit [6] and give natural extensions of the results of that paper. We start with the

DEFINITION 5.1. If $\Pi_{i=1}^{s} p_{i}^{a_{i}}$ is the prime decomposition of positive integer $k$ then for non-zero $m_{1}, m_{2}, \ldots$,

$$
\begin{gathered}
\omega_{1}\left(m_{1}, k\right)=\prod_{i=1}^{s}\left(\begin{array}{c}
1 /\left(1-p_{i}^{-m_{1}}\right) \\
a_{i}
\end{array}\right), \\
\omega_{2}\left(m_{1}, m_{2}, k\right)=\prod_{i=1}^{s}\left(\begin{array}{c}
\left.\left.1 /\left(1-p_{i}^{-m_{1}}\right)\left(1-p_{i}^{-m_{2}}\right)\right)\right), \\
a_{i}
\end{array}\right),
\end{gathered}
$$

and generally 


$$
\omega_{n}\left(m_{1}, m_{2}, \ldots, m_{n}, k\right)=\prod_{i=1}^{s}\left(\begin{array}{c}
\left.1 /\left(1-p_{1}^{-m_{1}}\right)\left(1-p_{t}^{-m_{2}}\right) \ldots\left(1-p_{t}^{-m_{n}}\right)\right) \\
a_{1}
\end{array}\right) .
$$

In [6] the function from (5.1) was shown to naturally occur in the Dirichlet series resulting from (1.4), (1.5), and (1.6). For example, (1.6) is equivalent to the statement

$$
\prod_{\substack{20, k=1 \\(, k)=1}} \zeta(j m+k n)^{-1 / k}=\sum_{k=1}^{\infty} \frac{\lambda(k) \omega_{1}(m, k)}{k^{n}}, \operatorname{Re} m \text { and } \operatorname{Re} n>1 \text {. }
$$

In this $\lambda(k)=\Pi_{i-1}^{s}(-1)^{a}$ is Liouville's arithmetic function (for an account of this function see Chandrasekharan [10]).

Given the results of our present paper, we can derive Dirichlet series generating functions for the multiplicative functions of Definition 5.1. Firstly applying the product operator

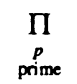

to each side of the identities in Corollaries 3.1 and 3.2, we have, letting each variable, say $x$ be replaced by $p^{-x}$ before employing the product operator,

THEOREM 5.2. If $R e m_{i}$ and $\operatorname{Re} n>1$ then

$$
\begin{gathered}
\prod_{\substack{a \geq 0, b \geq 1 \\
(a, b)=1}} \zeta\left(a m_{1}+b n\right)^{-1 / b}=\sum_{k=1}^{\infty} \frac{\lambda(k) \omega_{1}\left(m_{1}, k\right)}{k^{n}}, \\
\prod_{\substack{a, b=0 ; c<1 \\
(a, b, c)=1}} \zeta\left(a m_{1}+b m_{2}+c n\right)^{-1 / c}=\sum_{k=1}^{\infty} \frac{\lambda(k) \omega_{2}\left(m_{1}, m_{2}, k\right)}{k^{n}}, \\
\prod_{\substack{a, b, c=0, d=1 \\
(a, b, c, d)=1}} \zeta\left(a m_{1}+b m_{2}+c m_{3}+d n\right)^{-1 / d}=\sum_{k=1}^{\infty} \frac{\lambda(k) \omega_{3}\left(m_{1}, m_{2}, m_{3}, k\right)}{k^{n}},
\end{gathered}
$$

and generally

$$
\prod_{\substack{a_{a} \geq 0 ; a \geq 1 \\\left(a_{1}, a_{2}, \ldots, a_{h}, a\right)-1}} \zeta\left(a_{1} m_{1}+a_{2} m_{2}+\ldots+a_{h} m_{h}+a n\right)^{-1 / a}=\sum_{k=1}^{\infty} \frac{\lambda(k) \omega_{h}\left(m_{1}, m_{2}, \ldots, m_{h}, k\right)}{k^{n}} .
$$

PROOF. (5.5), as mentioned earlier, was given in [7]. To outline the proof we will briefly follow the logical path from (3.2) to (5.6). We choose this example as it allows the reader to see what is taking place, rather than presenting a multivariate generalized case such as the path from (3.5) to (5.8). Replacing $x, y$ and $z$ respectively by $p^{-m_{1}}, p^{-m_{2}}$, and $p^{-n}$ where $p$ is prime, then forming the product over the primes on both sides gives

$$
\prod_{\substack{a, b<0 ; c<=1 \\(a, b, c)=-1}} \prod_{p}\left(1-p^{-\left(a m_{1}+b m_{2}+c n\right)}\right)-\prod_{p}\left(1-p^{-n}\right)^{1 /\left(\left(1-p-m_{1}^{-1}\right)\left(1-p-m^{-2}\right)\right)},
$$

which is equivalent to

$$
\prod_{\substack{a, b \geq 0, c \geq 1 \\(a, b, c)=1}} \zeta\left(a m_{1}+b m_{2}+c n\right)^{-1 / c}=\prod_{p}\left(1-p^{-n}\right)^{1 /\left(\left(1-p-m^{-1}\right)\left(1-p-m^{-2}\right)\right)} .
$$

From here we simply expand the right side of (5.9) into the appropriate Dirichlet series leading to (5.6). This method can be just as well applied to the other identities in Corollary 5.2. End of proof.

Identities such as (5.9) can be written down immediately from many of the identities already presented here; for example (4.36) implies 


$$
\begin{aligned}
& \Pi_{\substack{a, b, c<d \\
(a, b, c, d)=1 \\
a, b, c) \geq 0, d<1}} \zeta\left(a m_{1}+b m_{2}+c m_{3}+d n\right)^{-1 / d}=\prod_{p}\left(\frac{\left(1-p^{-n}\right)\left(1-p^{-\left(m_{1}+m_{2}+n\right)}\right)\left(1-p^{-\left(m_{1}+m_{3}+n\right)}\right)}{\left(1-p^{-\left(m_{1}+n\right)}\right)\left(1-p^{-\left(m_{2}+n\right)}\right)\left(1-p^{-\left(m_{3}+n\right)}\right)}\right. \\
& \\
& \left.\frac{\left(1-p^{-\left(m_{2}+m_{3}+n\right)}\right)}{\left(1-p^{-\left(m_{1}+m_{2}+m_{3}+n\right)}\right)}\right)^{\sqrt{\left.(1-p)-m_{1}\right)\left(1-p-m_{2}\right)(1-p)}} ;
\end{aligned}
$$

valid for real parts of the following: $n, m_{1}+n, m_{2}+n, m_{3}+n, m_{1}+m_{2}+n, m_{1}+m_{3}+n, m_{2}+m_{3}+n$, $m_{1}+m_{2}+m_{3}+n$, all greater than unity. Also $m_{1}, m_{2}, m_{3}, n$ are $\approx 0$.

In [6], summations such as (5.5) were given some interpretation, showing that our function $\omega_{1}(m, k)$ occurs naturally in each of the companion identities (1.4), (1.5), (1.6). In order to properly find interpretations of the "prime products" presented it is necessary to use the

THEOREM 5.3. If $\operatorname{Re}(j m+k n)>1$ for positive integer $k$ then

$$
\zeta(j m+k n)^{1 / k}=\sum_{r=1}^{\infty} \frac{f_{j, k}(r)}{r^{j m+k n}},
$$

then with $\lambda(r)$ the Liouville function,

$$
f_{,, k}(r)=\prod_{t=1}^{s}(-1)^{a_{i}}\left(\begin{array}{c}
-1 / k \\
a_{t}
\end{array}\right)=\lambda(r) \prod_{t=1}^{s}\left(\begin{array}{c}
-1 / k \\
a_{t}
\end{array}\right)
$$

where $\prod_{i=1}^{s} p_{i}^{a_{i}}$ is the prime decomposition of positive integer $r$.

For a proof of this see [6], however the proof is elementary and based on nothing more than the binomial theorem and the Euler product form of the Riemann zeta function. Theorem 5.3 holds an important key to interpreting many of the identities of our present work. It is clear from (5.12) that $f_{j, k}(r)$ is really only a function of type $f_{k}(r)$, and so for instance the function under the product operation on left side of $(5.10)$ is

$$
\zeta\left(a m_{1}+b m_{2}+c m_{3}+d n\right)^{-1 / d}=\sum_{r=1}^{\infty} \frac{f_{-d}(r)}{a m_{1}+b m_{2}+c m_{3}+d n} .
$$

In applying the methods of [6] to the higher dimensional cases we straight away run into formal difficulties unless we choose appropriate identities to examine. It seems that the identities of Corollaries 3.1 and 3.2 again arise, further supporting our assertion in $\$ 3$ that they represent a class of "natural cases" of Theorem 2.1. Although the $j$ of $f_{j, k}(r)$ in (5.12) is seen to be superfluous we shall find it useful to keep as a means of identifying that this function arises for the $\zeta(j m+k n)$ function. We will distinguish then between functions generated by $\zeta\left(j_{1} m+k n\right)$ and $\zeta\left(j_{2} m+k n\right)$. Indeed, for the higher dimensional cases we will introduce further parameters to any $f_{j, k}(r)$, making it say $f_{a, b, c, j, k}(r)$, when arising for $\zeta\left(a m_{1}+b m_{2}+c m_{3}+j m_{4}+k n\right)^{1 / k}$. This allows us to identify the source of the Dirichlet generated coefficients should they have same values as other coefficient functions in the products. At this point we give our theorem, which essentially equates Dirichlet coefficients of (5.8). We first will need to give a definition.

DEFINITION 5.4. Consider the lattice point arrived at from the origin by the vector $\left\langle a_{1}, a_{2}, \ldots, a_{h}\right\rangle$. Let $\mathbf{N}\left\langle\mathbf{a}_{1}, \mathbf{a}_{2}, \ldots, \mathbf{a}_{\mathrm{h}}\right\rangle$ be an arbitrary positive integer associated with this lattice point in $h$-space.

THEOREM 5.5. If $N-1$ and $N\left\langle\mathbf{a}_{1}, \mathbf{a}_{2}, \ldots, \mathbf{a}_{\mathrm{h}}\right\rangle$ are any positive integers: 


$$
\begin{aligned}
\mu(N) \lambda(N) \omega_{h}\left(m_{1}, m_{2}, \ldots, m_{h}, N\right) & \\
= & \prod_{\substack{a_{1}, a_{2}, a_{h-1} \geq 0 \\
a_{h} \geq 1 \\
\left(a_{1}, a_{2}, \ldots, a_{h}\right)-1}} f_{a_{1}, a_{2}, \ldots, a_{h}}\left(\mathbf{N}\left\langle\mathbf{a}_{1}, \mathbf{a}_{2}, \ldots, \mathbf{a}_{\mathbf{h}}\right)\right) \mathbf{N}\left\langle\mathbf{a}_{1}, \mathbf{a}_{2}, \ldots, \mathbf{a}_{\mathbf{h}}\right\rangle^{-\left(a_{1} m_{1}+\ldots+a_{h-1} m_{h-1}\right)}
\end{aligned}
$$

with the summation over all solutions of

$$
N=\prod_{\substack{a_{1}, a_{2}, \quad a_{h-1} \geq 0 \\ a_{h} \geq 1 \\\left(a_{1}, a_{2}, \ldots, a_{h}\right)=1}} \mathrm{~N}\left\langle\mathbf{a}_{1}, \mathbf{a}_{2}, \ldots, \mathbf{a}_{\mathbf{h}}\right\rangle^{a_{h}}
$$

where all but a finite number of the $\mathbf{N}\left\langle\mathbf{a}_{1}, \mathbf{a}_{2}, \ldots, \mathbf{a}_{\mathbf{h}}\right\rangle$ are unity and only the non-unity factors are considered for $(5.14)$ ( $\mu$ is the Mobius function).

PROOF. This essentially parallels the proof of the analogous result given in [6]. We start from the finite product on the integer sequence $\left(\alpha_{i}\right), i \geq 1,1 \leq j \leq h$,

$$
\begin{aligned}
& \prod_{t=1}^{s} \zeta\left({ }_{1} \alpha_{t} m_{1}+{ }_{2} \alpha_{t} m_{2}+\ldots+{ }_{h-1} \alpha_{i} m_{h-1}+{ }_{h} \alpha_{t} m_{h}\right)^{1 / \alpha_{t}}= \\
& \qquad \sum_{r_{1} \geq 1} \sum_{r_{2} \geq 1} \ldots \sum_{r_{s} \geq 1} \prod_{t=1}^{s} f_{1} \alpha_{t}, \ldots h{ }_{t}\left(r_{t}\right) r_{t}{ }^{-\left(1, \alpha_{t} m_{1}+\ldots+{ }_{h-1} \alpha_{t} m_{h-1}+{ }_{h} \alpha_{t} m_{h}\right)}
\end{aligned}
$$

This is easily proved by induction on $s$. All partial products on the right side of (5.14) are of this kind since the lattice points in the hyperquadrant concerned are countable (recall our proof of Lemma 2.3 in §2). If $k$ is any positive integer the coefficient of $k^{-m_{h}}$ in (5.16) is determined from those terms $r_{i}{ }^{-\alpha_{i}}$ which are factors of $k^{-1}$. In other words, the Dirichlet generated coefficient of $k^{-m_{k}}$ in

$$
\sum \prod_{i=1}^{s} f_{{ }_{1} \alpha_{i}, \ldots, h} \alpha_{i}\left(r_{i}\right) r_{i}^{-\left(\alpha_{i} m_{1}+\cdots++_{h-1} \alpha_{i} m_{h}\right)},
$$

where the summation is over each solution of $k=\Pi_{i-1}^{s} r_{t}^{h^{\alpha}}$.

In (5.16) we may choose the $\alpha$ sequence to conform with the lattice points in the product operators of (5.14) and (5.15), owing to the countability of the lattice points in this "rayed from origin" region. Hence the product operator of (5.17) transforms into that of (5.14) and (5.15), and we can allow $s$ to increase indefinitely. The Mobius function on the left side of (5.14) is due to our considering the reciprocal identity of (5.8) in order to better apply (5.11) to the analysis. This is the formal proof. The stipulation in the theorem that "all but a finite number of the $N\left\langle a_{1}, a_{2}, \ldots, a_{h}\right\rangle$ are unity" simply means that, whilst any term under the product operator may be non-unity, be it the millionth term or the first term in the partial product, each product under the summation in (5.14) is considered finite. Of course the left side of (5.14) comes from the reciprocal identity produced from (5.8) using Mobius inversion. End of proof.

It is important to understand that Theorem 5.5 is essentially a multidimensional factorization theorem where each visible lattice point in the region is allowed an arbitrary positive integer value raised to a certain power, and all possible products of such numbers are considered. If in Theorem 5.5 we take the example where $N$ is a prime, the complexity reduces to a simple result. (5.14) is basically a multidimensional Dirichlet summation representation of the arithmetic function on its left side. The case where $h=2$ of Theorem 5.5 was given as part of a theorem in [6].

\section{CONCLUDING REMARKS}

One of the main issues confronting the author has been to select salient results from the apparently unending mass of results. Trying to determine or guess what is most natural is not easy, so an attempt was made to give the results which appeared most striking. The careful reader will by now appreciate 
that a full classification of the identities herein would amount to a classification of symmetric rotations in arbitrary dimensional lattices; not a simple matter. Nevertheless, the vpv idea exemplified in Lemma 2.3 is applicable to any rayed-from-origin Euclidean region. We have shown that classes of interdependent vpv identities exist probably in any dimension, and there appears to be a variety of methods for obtaining these. A consistent theoretical approach to classification of "companion identities" would seem desirable at this stage, given that we have now opened the door to a sensible multivariate world.

In this paper we have refrained from entering deeply into particular logarithmic derivatives of the products. Also we have not fully explored the interesting convergence boundary cases of many results. We have not yet followed up higher dimensional analogues in the more general sense of Theorems 1.1 and 1.2 , which lead us to numerous results on Ramanujan type arithmetic functions on the one hand, and Jacobi theta function results on the other.

Future work on vpv identities may involve lattice sums of chemistry as presented in Glasser and Zucker [15], applications to the theory of partitions, or some of the vpv work may find application in additive number theory. This latter seems a natural application since additive functions are invariant under some of the transforms in this paper. As such, vpv methods may be linked to some of the problems given in Elliott [13, pp. 330-341].

ACKNOWLEDGEMENT. The author is indebted to: Professor R. J. Baxter, who kindly arranged Visitor Status and access at Australian National University; Professor G. E. Andrews, whose thoughtful and positive response has been a catalyst; Dr. Peter Forrester who was always in the right mathematical place, besides being a source of encouragement.

\section{REFERENCES}

[1] ANDREWS, G. E. (1976). The Theory of Partitions, Vol. 2, Encyclopedia of Mathematics and its Applications, Addison-Wesley.

[2] APOSTOL, T. M. (1976). Introduction to Analytic Number Theory, Springer-Verlag, New York.

[3] BERNDT. B. C. (1985). Ramanjan's Notebooks, Part I, Springer-Verlag, New York.

[4] CAMPBELL, G. B. (1993). A new class of infinite products, and Euler's totient, Int. J. of Math. and Math. Sci. (to appear).

[5] CAMPBELL, G. B. (1993). Dirichlet summations and products over primes, Int. J. of Math. and Math. Sci., Vol. 16, No. 2, 359-372.

[6] CAMPBELL, G. B. (1992). Multiplicative functions from Riemann zeta function products, J. Ramanujan Soc. 7, No. 1, 51-62.

[7] CARLITZ, L. (1963). Some generating functions, Duke Math. J. 30, 191-201.

[8] CARLITZ, L. (1963). A problem in partitions, Duke Math. J. 30, 203-213.

[9] CARLITZ, L. and ROSELLE, D. P. (1966). Restricted bipartite functions, Pacific J. Math. 19, 221-228.

[10] CHANDRASEKHARAN, K. (1970). Arithmetical Functions, Vol. 67, Springer-Verlag, New York.

[11] CHEEMA, M. S. (1964). Vector partitions and combinatorial identities, Math. Comp. 18, 414-420.

[12] CHEEMA, M. S. and MOTZKIN, T. S. (1971). Multipartitions and multipermutations, Proc. Symp. Pure Math. 19, 37-39.

[13] ELLIOTT, P. D. T. A. (1980). Probabilistic Number Theory II, Vol. 240, Springer-Verlag, New York.

[14] FRANKEL, N. E.; GLASSER, M. L.; HUGHES, B. D.; and NINHAM, B. W. (1992). J. Phys. A, 985 (to appear).

[15] GLASSER, M. L. and ZUCKER, I. J. (1980). Lattice Sums, Theoretical Chem.: Adv. Persp. Vol. 5, 67-139.

[16] LEWIN, L. (1981). Polylogarithms and Associated Functions, North-Holland, New York.

[17] LEWIN, L. (1982). The dilogarithm in algebraic fields, J. Austral. Math. Soc. Ser. A, 33, 302-330. 


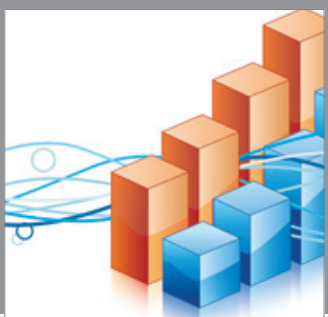

Advances in

Operations Research

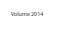

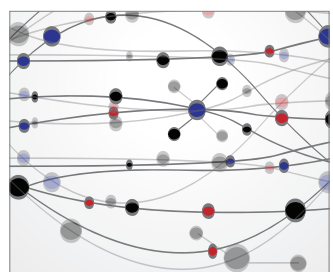

\section{The Scientific} World Journal
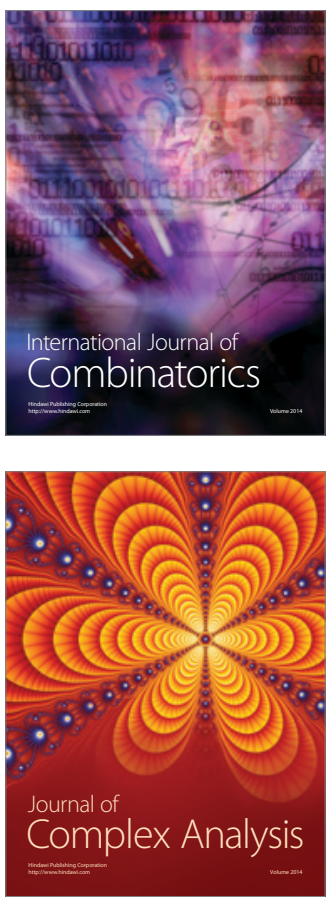

International Journal of

Mathematics and

Mathematical

Sciences
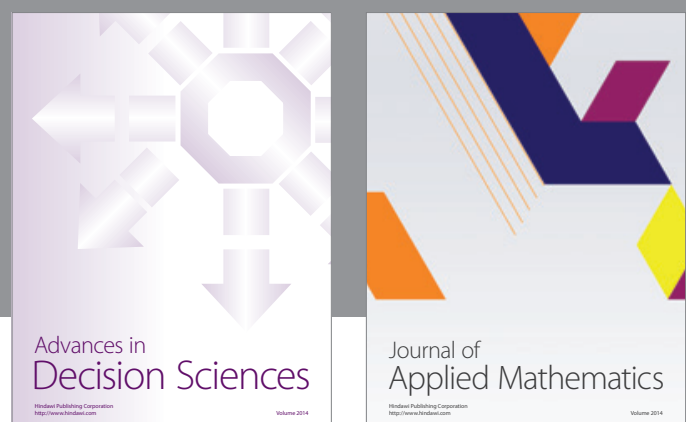

Journal of

Applied Mathematics
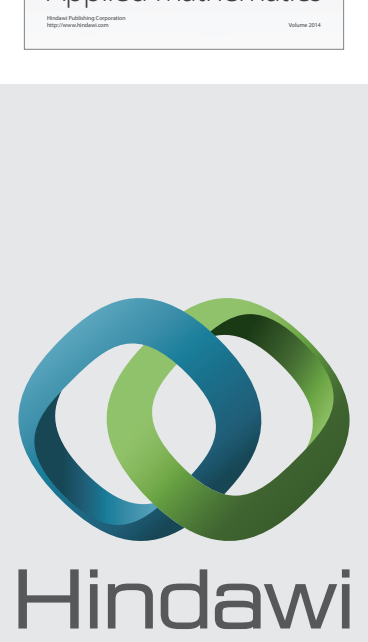

Submit your manuscripts at http://www.hindawi.com
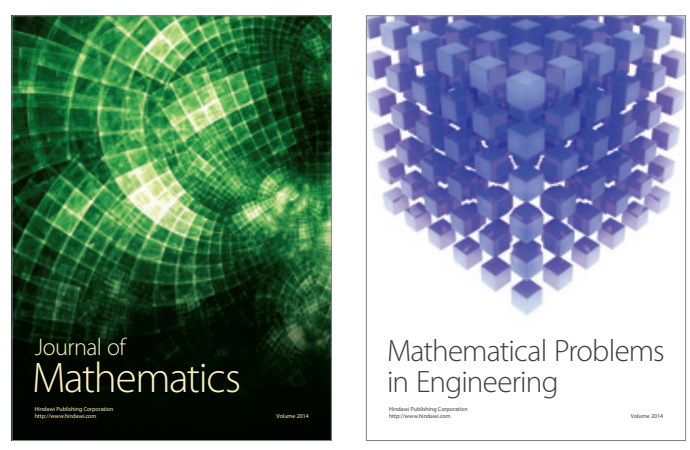

Mathematical Problems in Engineering
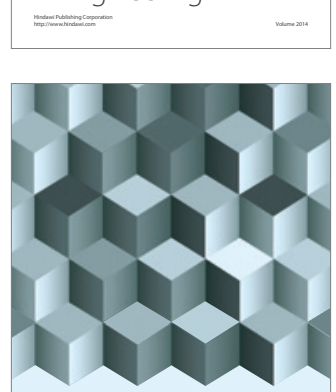

Journal of

Function Spaces
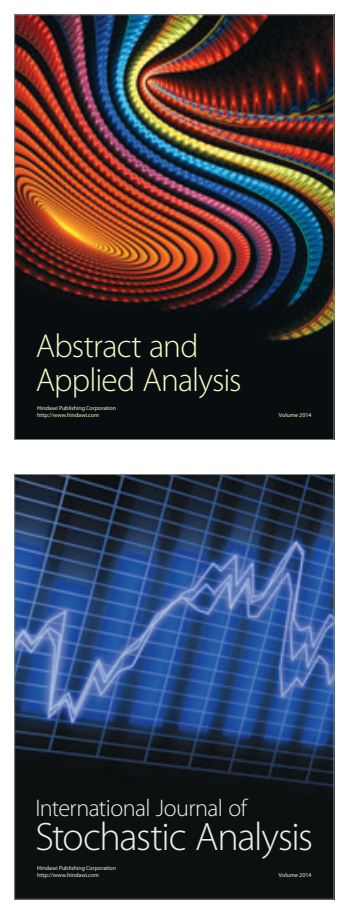

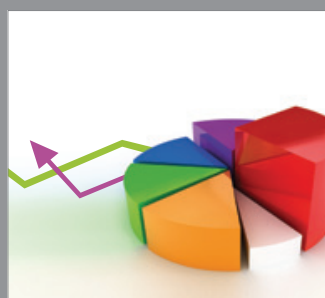

ournal of

Probability and Statistics

Promensencen
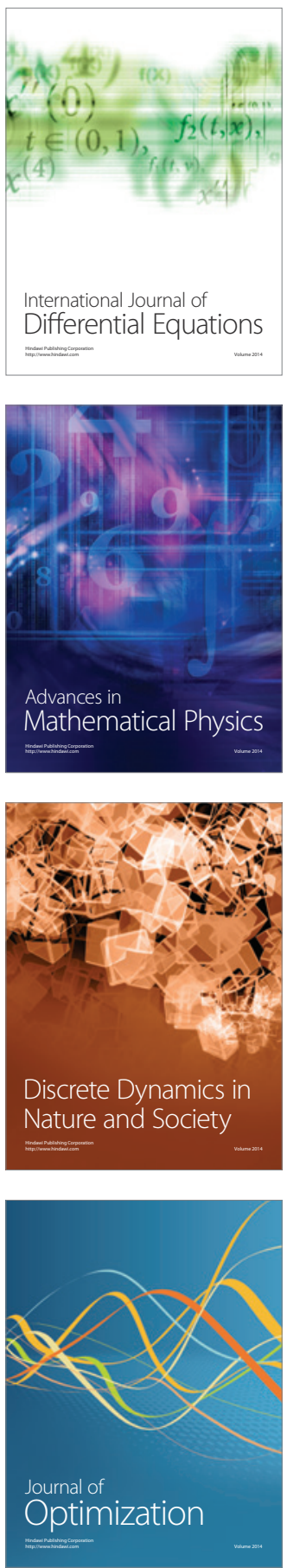